\title{
Some results on random design regression with long memory errors and predictors
}

\author{
Rafał Kulik \\ Department of Mathematics \& Statistics, University of Ottawa \\ 585 King Edward Avenue, Ottawa ON K1N 6N5, Canada \\ email: rkulik@uottawa.ca
}

Paweł Lorek

Mathematical Institute, University of Wrocław

pl. Grunwaldzki 2/4, 50-384 Wrocław, Poland

email: lorek@math.uni.wroc.pl

June 14, 2010

\begin{abstract}
This paper studies nonparametric regression with long memory (LRD) errors and predictors. First, we formulate general conditions which guarantee the standard rate of convergence for a nonparametric kernel estimator. Second, we calculate the Mean Integrated Squared Error (MISE). In particular, we show that LRD of errors may influence MISE. On the other hand, an estimator for a shape function is typically not influenced by LRD in errors. Finally, we investigate properties of a data-driven bandwidth choice. We show that Averaged Squared Error (ASE) is a good approximation of MISE, however, this is not the case for a cross-validation criterion.
\end{abstract}

\section{Introduction}

Consider the random design regression model,

$$
Y_{i}=m\left(X_{i}\right)+\varepsilon_{i}, \quad i=1, \ldots, n,
$$

where $X_{i}, \varepsilon_{i}, i=1, \ldots, n$, are two mutually independent sequences of random variables. We investigate the problem of estimating function $m(\cdot)$. This problem is quite well understood for weakly dependent data. Also, in the last two decades, it has received a lot of attention in case of long range dependence (LRD). In this situation most of results have been obtained under quite specific assumptions on the errors and/or predictors. In particular, it is typically 
assumed that both sequences are infinite order moving averages, or they are defined as (nonlinear) functionals of Gaussian sequences. However, in the recent years different authors proposed many new (nonlinear) LRD models. Although it is reasonable to assume some structure on the observable predictors, particular assumptions on the errors are almost impossible to verify. Therefore, one of our goals is to state general conditions, which guarantee appropriate limit theorems.

In Section 2.2 we discuss central limit theorem for the Nadaraya-Watson estimator of $m(\cdot)$. As it is well known, for LRD data we have a dichotomous behaviour: if a bandwidth $h$ is small, then the rate of convergence is $\sqrt{n h}$, the same as in i.i.d. case. Otherwise, if bandwidth is large, long memory contributes. We refer the reader to [17] for the most general result; see also [4], [6], [7]. We state general conditions, which guarantee $\sqrt{n h}$ rate of convergence. These conditions can be easily verified for (subordinated) linear LRD processes, FARIMA-GARCH models, stochastic volatility models (including LARCH), as well as for antipersistent errors. In particular, if $\varepsilon_{i}, i \geq 1$, is a linear process such that $\operatorname{Var}\left(\sum_{i=1}^{n} \varepsilon_{i}\right) \sim C n^{2-\alpha}, \alpha \in(0,1)$, then $\sqrt{n h}$-consistency holds if $h n^{1-\alpha} \rightarrow 0$. This agrees with previous results. (Here and in the sequel, $C$ is a generic constant). On the other hand, however, if the errors are described by a stochastic volatility, then $\sqrt{n h}$-consistency always holds.

To verify that the condition $h n^{1-\alpha} \rightarrow 0$ holds, we need to know the parameter $\alpha$. However, random variables $\varepsilon_{i}, i=1, \ldots, n$, are not directly observable, and a performance of various estimators of $\alpha$ is not clear. Therefore, we will modify our estimation problem. In some cases, for a purpose of an exploratory data analysis, it suffices to estimate a shape function, $m^{*}(x)=m(x)-\int m f$. As indicated in [8], [20] and [16], LRD of errors does not influence estimation of $m^{*}(\cdot)$. This effect is proven here for the Nadaraya-Watson estimator. In fact, for the linear LRD processes mentioned above, we obtain $\sqrt{n h}$-consistency if the predictors are independent and $h^{5} n^{1-\alpha} \rightarrow 0$, which is much weaker then the previous condition. However, this approach does not work in case of LRD predictors.

Next, we investigate properties of the Mean Integrated Squared Error (MISE). We assume that $\varepsilon_{i}, i \geq 1$, is the linear process as mentioned above. We show that the optimal MISE has a dichotomous behaviour: if the memory parameter $\alpha$ is greater than $4 / 5$, then the rate of convergence is $n^{-4 / 5}$, the same as in for i.i.d. errors. However, if $\alpha<4 / 5$, then the rate of convergence is $n^{-\alpha}$. Interestingly, a possible LRD of predictors does not influence the asymptotic behaviour of MISE. Similar results were obtained for density estimation, see [10]. On the other hand, in a fixed-design case, LRD always influences the rates of convergence. For details we refer to [11].

To reduce the influence of LRD on MISE, we may consider the shape function. It is shown that for independent predictors, the Mean Integrated Squared Error corresponding to $m^{*}$, has the same asymptotic behaviour as in case of i.i.d. errors. In other words, from expected-risk point of view, long memory 
does not influence estimation of the shape function. We also note in passing that the optimal bandwidth choice for the shape estimation agrees with the optimal one for the estimation of the original function, as long as $\alpha>2 / 5$.

With help of formulas for MISE, we obtain the optimal bandwidths. As usual, they are not quite practical, since they involve unknown parameters and a data-driven method has to be used. As argued in [12], a plug-in method has some advantages over cross-validation. However, let us note that the optimal bandwidth is of the form $C n^{-1 / 5}$ if $\alpha>2 / 5$, and $C n^{-(1-\alpha) / 3}$, otherwise. Consequently, we have to know $\alpha$ to be able to construct an appropriate plug-in bandwidth selector. Therefore, we focus on cross-validation. Let us indicate first that cross-validation is the valid procedure in a fixed-design case. The procedure produces a bandwidth which is close to the optimal one, and cross-validation criterion itself is a good approximation to MISE. The reader is referred to [12]. In the density estimation case, however, cross-validation is a good approximation to MISE if and only if $\alpha>4 / 5$, see [13] and [5] as well as discussion in Section 2.4 for more details.

We will show that for random-design regression, the empirical minimizer of the cross-validation criterion is a good approximation to the optimal $h$, however, the cross-validation itself is the valid procedure for $\alpha>4 / 5$ (which agrees with findings in [13] and [5]).

The paper is organized as follows. In Section 2.1 we collect assumptions and define estimators. Sections 2.2, 2.3 and 2.4 contain results on central limit theorem, mean square error and bandwidth choice, respectively. In Section 3 we

illustrate our findings by simulations. Next, in Section 4 we present examples of time series, where it is possible to verify our conditions. Finally, the proofs are presented in the last section.

\section{Results}

\subsection{Assumptions and estimators}

We will consider the following assumptions on the predictors $X_{i}, i \geq 1$ :

(P1) $X_{i}, i \geq 1$, is a sequence of i.i.d. random variables. In this case, let $\mathcal{X}_{i}=\sigma\left(X_{i}, \ldots, X_{1}\right)$

(P2) $X_{i}, i \geq 1$, is the infinite order moving average

$$
X_{i}=\sum_{k=0}^{\infty} a_{k} \zeta_{i-k}, \quad a_{0}=1,
$$

where $\zeta_{i},-\infty<i<\infty$, is the sequence of centered, i.i.d. Gaussian random variables and for $\alpha_{X} \in(0,1), a_{k}=A_{0} k^{-\left(\alpha_{X}+1\right) / 2}$ for some $A_{0}$. 
Consequently, $X_{i}$ are Gaussian and they are assumed to have unit variance. In this case we denote $\mathcal{X}_{i}=\sigma\left(\zeta_{i}, \zeta_{i-1}, \ldots\right)$. Furthermore, note that $\operatorname{Var}\left(\sum_{i=1}^{n} X_{i}\right) \sim A_{1}^{2} n^{2-\alpha_{X}}$, where $A_{1}$ is a finite constant.

We note that from the point of view of our results stated below, (P1) can be treated as the special case of (P2), by plugging-in $\alpha_{X}=1$. Thus, the results which are stated under (P2) assumption are valid also under (P1).

Under (P1), we do not need to assume a particular structure of errors $\varepsilon_{i}$, $i \geq 1$. The general assumption is

(E0) $\varepsilon_{i}=G\left(\eta_{i}, \eta_{i-1}, \ldots\right), i \geq 1$, where $\eta_{i},-\infty<i<\infty$ is an i.i.d. sequence, independent of $X_{i}, i \geq 1$. We also assume that $\mathrm{E}\left[\varepsilon_{1}\right]=0, \mathrm{E}\left[\varepsilon_{1}^{2}\right]=1$. It assures that $\varepsilon_{i}, i \geq 1$, is a stationary ergodic sequence. Denote $\mathcal{H}_{i}=$ $\sigma\left(\eta_{i}, \eta_{i-1}, \ldots\right)$.

Under (P2) we will assume that

(E2) $\varepsilon_{i}, i \geq 1$, is the infinite order moving average

$$
\varepsilon_{i}=\sum_{k=0}^{\infty} c_{k} \eta_{i-k}, \quad c_{0}=1
$$

where $\eta_{i},-\infty<i<\infty$, is the sequence of centered, i.i.d. random variables with a finite fourth moment, $\mathrm{E}\left[\varepsilon_{1}^{2}\right]=1$, independent of $X_{i}, i \geq 1$. We also assume that for $\alpha \in(0,1), c_{k} \sim C_{0} k^{-(\alpha+1) / 2}$, as $k \rightarrow \infty$. Then $\operatorname{Var}\left(\sum_{i=1}^{n} \varepsilon_{i}\right) \sim C_{1}^{2} n^{2-\alpha}$, where $C_{1}$ is a constant.

We consider the classical Nadaraya-Watson estimator

$$
\hat{m}(x)=\hat{m}_{h}(x)=\frac{1}{n h} \frac{1}{\hat{f}_{h}(x)} \sum_{i=1}^{n} Y_{i} K\left(\frac{x-X_{i}}{h}\right),
$$

where a nonnegative and bounded kernel $K$ fulfills the usual conditions:

$$
\int K(u) d u=1, \quad \int u K(u) d u=0, \quad \kappa_{2}:=\int u^{2} K(u) d u .
$$

For a future use we denote $K_{h}(\cdot)=K(\cdot / h)$ and $\kappa_{1}:=\int K^{2}(u) d u \neq 0$. The bandwidth $h=h_{n}$ fulfills the usual conditions $h \rightarrow 0$ and $n h \rightarrow \infty$. Furthermore, $\hat{f}_{h}(x)$ is the standard kernel estimator of the density $f$ of $X_{1}$.

Define further the shape function

$$
m^{*}:=m-\int m f
$$

and its estimator $\widehat{m^{*}}=\hat{m}-\widehat{\int m f}$, where

$$
\widehat{\int m f}:=\frac{1}{n} \sum_{i=1}^{n} Y_{i} .
$$


Note that the latter is the unbiased estimator of $\int m f$, i.e.

$$
\frac{1}{n} \sum_{i=1}^{n} \mathrm{E}\left[Y_{i}\right]=\mathrm{E}\left[m\left(X_{1}\right)\right]=\int m f .
$$

Finally, we will assume that $f$ and $m$ are twice differentiable with bounded derivatives and that $f(x)>0$ for each $x$.

\subsection{Central limit theorems}

Throughout this section we assume that (E0) holds. Let us formulate the following conditions:

$$
\begin{gathered}
\frac{\sqrt{n h}}{n} \sum_{i=1}^{n}\left(\mathrm{E}\left[\varepsilon_{i} \mid \mathcal{H}_{i-1}\right]-\varepsilon_{i}\right)=o_{P}(1) . \\
\frac{\sqrt{n h}}{n} \sum_{i=1}^{n} \mathrm{E}\left[\varepsilon_{i} \mid \mathcal{H}_{i-1}\right]=o_{P}(1) . \\
\frac{\sqrt{n h} h^{2}}{n} \sum_{i=1}^{n} \mathrm{E}\left[\varepsilon_{i} \mid \mathcal{H}_{i-1}\right]=o_{P}(1) . \\
h^{5} n^{1-\alpha_{X}} \rightarrow 0 . \\
h n^{1-\alpha_{X}} \rightarrow 0 .
\end{gathered}
$$

Define

$m_{n}(x):=\mathrm{E}\left[K\left(\left(x-X_{1}\right) / h\right) m\left(X_{1}\right)\right] / \mathrm{E}\left[K\left(\left(x-X_{1}\right) / h\right)\right], \quad m_{n}^{*}(x)=m_{n}(x)-\int m f$.

Clearly, the bias is

$$
\operatorname{bias}(x)=m_{n}(x)-m(x) \sim h^{2}\left(\frac{m^{\prime \prime}(x)}{2}+\frac{m^{\prime}(x) f^{\prime \prime}(x)}{f(x)}\right) \int u^{2} K(u) d u .
$$

Proposition 2.1. Assume (P1) and (E0). Under the conditions (A) and (B1) we have

$$
\sqrt{\frac{n h}{\kappa_{1}}} \sqrt{\hat{f}(x)}\left(\hat{m}(x)-m_{n}(x)\right) \stackrel{\mathrm{d}}{\rightarrow} N(0,1) .
$$

Assume (P2) and (E2). Under the conditions (A), (B1) and (C1), the asymptotics (3) holds.

Remark 2.2. Note that there is no symmetry between LRD assumptions on $\varepsilon_{i}$ and $X_{i}$. For example, assume that $\varepsilon_{i}$, and thus $\mathrm{E}\left[\varepsilon_{i} \mid \mathcal{H}_{i-1}\right]$ is the linear process with $\operatorname{Var}\left(\sum_{i=1}^{n} \mathrm{E}\left[\varepsilon_{i} \mid \mathcal{H}_{i-1}\right]\right) \sim C_{1}^{2} n^{2-\alpha}, \alpha \in(0,1)$; see Example 4.1. Then (B1) holds if $h n^{1-\alpha} \rightarrow 0$, whereas the assumption for $\alpha_{X}$ requires $h^{5} n^{1-\alpha_{X}} \rightarrow 0$. 
Proposition 2.3. Assume (P2). Under the conditions (A), (B2) and (C2) we have

$$
\sqrt{\frac{n h}{\kappa_{1}}} \sqrt{\hat{f}(x)}\left(\widehat{m^{*}}(x)-m_{n}^{*}(x)\right) \stackrel{\mathrm{d}}{\rightarrow} N(0,1) .
$$

Remark 2.4. The result under (P1) follows by taking $\alpha_{X}=1$ in (C2). In this case the condition is always fulfilled. The difference between the estimators of $m(\cdot)$ and $m^{*}(\cdot)$ appears by comparison of (B1) and (B2). In case of $m^{*}(\cdot)$ much larger bandwidths are allowed to achieve classical rates of convergence. The additional condition (A), required for $m^{*}(\cdot)$ estimation is typically easy to verify, even for long memory or non-stationary sequences.

Recall from the previous remark that LRD in predictors basically does not matter. This is not the case for the shape estimation. Assume that $h n^{1-\alpha_{X}} \rightarrow$ $\infty$, but (A), (B2), (C1) and

$$
\frac{\sqrt{n h}}{n^{\alpha X} / 2} \frac{1}{n} \sum_{i=1}^{n} \mathrm{E}\left[\varepsilon_{i} \mid \mathcal{H}_{i-1}\right]=o_{P}(1)
$$

hold. For example, this happens when $\varepsilon_{i}$, and thus $\mathrm{E}\left[\varepsilon_{i} \mid \mathcal{H}_{i-1}\right]$ is the linear process with $\operatorname{Var}\left(\sum_{i=1}^{n} \mathrm{E}\left[\varepsilon_{i} \mid \mathcal{H}_{i-1}\right]\right) \sim C_{1}^{2} n^{2-\alpha}, \alpha \in(0,1)$, and $h^{5} n^{1-\alpha} \rightarrow 0$. It follows from the proof of Proposition 2.3 that

$$
n^{\alpha_{X} / 2}\left(\widehat{m^{*}}(x)-m_{n}^{*}(x)\right) \stackrel{\mathrm{d}}{\rightarrow} \mathcal{N}\left(0, A_{1}^{2} \mathrm{E}^{2}\left[m\left(X_{1}\right) X_{1}\right]\right) .
$$

\subsection{Mean Square Error}

In this section we establish asymptotic formulas for mean integrated squared error for both $m(\cdot)$ and $m^{*}(\cdot)$. In particular, it will be shown that we may improve the rates of convergence, if we estimate the shape function instead of $m(\cdot)$.

Consider the following weighted versions of the mean integrated squared errors:

$$
\begin{aligned}
\operatorname{MISE}_{r}(h) & :=\int \mathrm{E}\left[\left(\hat{m}_{h}(x)-m(x)\right)^{2}\right] r(x) d x, \\
\operatorname{MISE}_{r}^{*}(h) & :=\int \mathrm{E}\left[\left(\hat{m}_{h}^{*}(x)-m^{*}(x)\right)^{2}\right] r(x) d x,
\end{aligned}
$$

where $r(\cdot)$ is a weight function and integrals are taken over a support of $f$.

Proposition 2.5. Assume (P2) and (E2). Then we have

$$
\begin{aligned}
\operatorname{MISE}_{r}(h) \sim & \frac{\kappa_{1}}{n h} \int \frac{r(x)}{f(x)} d x+\frac{h^{4} \kappa_{2}^{2}}{4} \int\left(\frac{m^{\prime \prime}(x) f(x)+2 m^{\prime}(x) f^{\prime}(x)}{f(x)}\right)^{2} r(x) d x \\
& +C_{1}^{2} n^{-\alpha}+C_{1}^{2} h^{2} \kappa_{2} n^{-\alpha} \int \frac{f^{\prime \prime}(x)}{f(x)} r(x) d x .
\end{aligned}
$$


Remark 2.6. The first term in $\operatorname{MISE}_{r}(h)$ describes i.i.d. type behaviour, the second one is due to bias. The terms involving $n^{-\alpha}$ describe a possible contribution of long memory. Note that we have to include the term $h^{2} n^{-\alpha}$. These terms do not have influence on the optimal behaviour of MISE, but they influence $h_{\mathrm{opt}}$, the optimal bandwidth choice. Indeed, one can verify that

$$
h_{\mathrm{opt}} \sim \begin{cases}C n^{-1 / 5} & \text { if } \alpha>2 / 5 \\ C n^{-(1-\alpha) / 3} & \text { if } \alpha<2 / 5\end{cases}
$$

so that $\operatorname{MISE}_{r}\left(h_{\mathrm{opt}}\right)$ is proportional to $n^{-4 / 5}$ if $\alpha>4 / 5$ and $n^{-\alpha}$, if $\alpha<4 / 5$. Note also that there is no contribution of LRD of the predictors.

Proposition 2.7. Assume that

$$
\operatorname{Var}\left(\sum_{i=1}^{n}\left(\mathrm{E}\left[\varepsilon_{i} \mid \mathcal{H}_{i-1}\right]-\varepsilon_{i}\right)\right)=O(n)
$$

holds. Under (P2) and (E2) we have

$$
\begin{aligned}
\operatorname{MISE}_{r}^{*}(h) & \sim \frac{\kappa_{1}}{n h} \int \frac{r(x)}{f(x)} d x \\
+ & \frac{h^{4}}{2} \int\left(\frac{m^{\prime \prime}(x) f(x)+2 m^{\prime}(x) f^{\prime}(x)}{f(x)}\right)^{2} r(x) d x+A_{1}^{2} \mathrm{E}^{2}\left[m\left(X_{1}\right) X_{1}\right] n^{-\alpha_{X}} .
\end{aligned}
$$

Remark 2.8. The condition (5) can be verified for many time series, including time series with long memory (see Section 4). The result under (P1) can be obtained by taking $\alpha_{X}=1$; then the last term is negligible. Consequently, under (P1) we remove long memory of errors, however, under (P2) there is an additional term due to long memory of predictors.

Under (P1) the optimal bandwidth, $h_{\mathrm{opt}}^{*}$, is proportional to $n^{-1 / 5}$, yielding $\operatorname{MISE}_{r}^{*}\left(h_{\text {opt }}^{*}\right) \sim C n^{-4 / 5}$.

Remark 2.9. Hall and Hart, [11], were the first who proved the mean squared error behaviour in case of fixed-design regression. The meaning of their results is that LRD in errors always influences estimation of the conditional mean.

On the other hand, in case of kernel density estimation based on LRD data $\varepsilon_{1}, \ldots, \varepsilon_{n}$, Hall and Hart [10] showed a similar dichotomous behaviour, as described in Proposition 2.5.

\subsection{Empirical bandwidth choice}

In this section we study properties of empirical bandwidth selector procedures. We shall focus on the case of i.i.d. predictors, to show an influence of LRD errors on the empirical risk. We will work also under the two additional assumptions: $\mathrm{E}\left[m^{2}\left(X_{1}\right)\right]<\infty$ and that $f$ has bounded support. This will simplify some computations and allow us to write $\operatorname{MISE}_{f}(h)$. Let

$$
\operatorname{ASE}(h)=\frac{1}{n} \sum_{i=1}^{n}\left(\hat{m}_{h}\left(X_{i}\right)-m\left(X_{i}\right)\right)^{2}
$$


be the Averaged Squared Error.

First, we answer the question, whether minimization of ASE leads to a valid minimizer. The answer is affirmative: the meaning of (6) is that the quotient of $\hat{h}$, the minimizer of ASE, and the minimizer of $\mathrm{MISE}_{f}$ converges to 1 in probability. Furthermore, $\operatorname{ASE}(\hat{h}) / \operatorname{MISE}\left(h_{\mathrm{opt}}\right) \stackrel{\mathrm{P}}{\rightarrow} 1$.

Proposition 2.10. Assume that $f, m \in \mathcal{C}^{2}$. Let $B_{1}<B_{2}$ be finite and positive constants. Under (P1) and (E2) we have uniformly over $\left[B_{1} h_{\mathrm{opt}}, B_{2} h_{\mathrm{opt}}\right]$,

$$
\operatorname{ASE}(h)-\operatorname{MISE}_{f}(h)=o_{P}\left(\operatorname{MISE}_{f}\left(h_{\mathrm{opt}}\right)\right) .
$$

However, what we are interested in from a practical point of view, is, first, whether a cross-validation produces a valid bandwidth, and, second, whether a cross-validation is a good approximation to ASE and MISE. To answer this, let $\hat{m}_{j, h}(\cdot)$ be the version of the estimator (1), where the summation is over $i \notin I_{j}(l)$, where $I_{j}:=\{i:|j-i|>l\}$. Empirical cross-validation bandwidth is obtained via minimizing

$$
\mathrm{CV}_{l}(h):=\frac{1}{n} \sum_{i=1}^{n}\left(Y_{i}-\hat{m}_{i, h}\left(X_{i}\right)\right)^{2} .
$$

Denote $\mathrm{CV}(h)=\mathrm{CV}_{0}(h)$. Note that if both predictors and errors are i.i.d., then

$$
\mathrm{E}[\mathrm{CV}(h)]=\mathrm{E}[\operatorname{ASE}(h)]+\mathrm{E}\left[\varepsilon_{1}^{2}\right]=\operatorname{MiSE}_{f}(h)+\mathrm{E}\left[\varepsilon_{1}^{2}\right],
$$

i.e. in the average sense $\mathrm{CV}(h)$ is the exact approximation of $\operatorname{MISE}_{f}(h)+\mathrm{E}\left[\varepsilon_{1}^{2}\right]$.

The result for LRD data is as follows.

Proposition 2.11. Assume that $f, m \in \mathcal{C}^{2}$. Let $B_{1}<B_{2}$ be finite and positive constants. Under (P1) and (E2) we have uniformly over $\left[B_{1} h_{\mathrm{opt}}, B_{2} h_{\mathrm{opt}}\right]$,

$$
\frac{\mathrm{CV}(h)-\operatorname{MISE}_{f}(h)-\frac{1}{n} \sum_{i=1}^{n} \varepsilon_{i}^{2}}{\operatorname{MISE}_{f}\left(h_{\mathrm{opt}}\right)}=\frac{1}{\operatorname{MISE}_{f}\left(h_{\mathrm{opt}}\right)} \frac{1}{n^{2}} \sum_{\substack{j, j^{\prime}=1 \\ j \neq j^{\prime}}}^{n} \varepsilon_{j} \varepsilon_{j^{\prime}}+o_{p}(1) .
$$

Let us comment on the above result. For a fixed-design case, under appropriate conditions on $l$, we have (see [12])

$$
\mathrm{CV}_{l}^{\prime}(h)-\operatorname{MISE}^{\prime}(h)-\frac{1}{n} \sum_{i=1}^{n} \varepsilon_{i}^{2}=o_{P}\left(n^{-4 \alpha / 5}\right),
$$

uniformly over $\left[C n^{-\alpha / 5}, C^{\prime} n^{-\alpha / 5}\right], C<C^{\prime}$. (Here, $\mathrm{CV}_{l}^{\prime}(h)$ and $\mathrm{MISE}^{\prime}(h)$ are defined in a slightly different way, to accommodate fixed-design). Note that $n^{-4 \alpha / 5}$ is the rate of $\operatorname{MISE}^{\prime}\left(h_{\mathrm{opt}}^{\prime}\right)$ and $n^{-\alpha / 5}$ is asymptotically proportional to $h_{\mathrm{opt}}^{\prime}$, where the latter is the asymptotically optimal bandwidth in the fixed 
design regression. This means that the ratio of the bandwidth obtained by cross-validation and the MISE optimal bandwidth converges to 1 in probability. Also, $\mathrm{E}\left[\mathrm{CV}_{l}^{\prime}\left(h_{\text {opt }}^{\prime}\right)\right]$ provides a valid approximation to $\operatorname{MISE}^{\prime}\left(h_{\text {opt }}^{\prime}\right)+\mathrm{E}\left[\varepsilon_{1}^{2}\right]$. (The last statement is intuitive only, since the rate at the right hand side of (7) is in probability rather than in $L^{1}$ ).

Now, from Proposition 2.11 we conclude that in the random-design regression $\hat{h}_{\mathrm{CV}}$, the minimizer of $\mathrm{CV}(h)$, has the property $\hat{h}_{\mathrm{CV}} / h_{\mathrm{opt}} \stackrel{\mathrm{P}}{\rightarrow} 1$. However, $\mathrm{CV}(h)$ itself provides a valid approximation only if $\alpha>4 / 5$. This agrees with the results in [13] in case of density estimation.

\section{$3 \quad$ Numerical studies}

Simulation studies were conducted as follows:

1. We set $h$.

2. Simulate 100 errors $\varepsilon_{i}$ from ARIMA with different LRD parameters $d$. Note that $d=(1-\alpha) / 2$ and i.i.d. case corresponds to $d=0$. We used R-package fracdiff.

3. Simulate 100 predictors following standard normal random variables. First, we simulate i.i.d. predictors, then LRD predictors with $d_{X}:=(1-$ $\left.\alpha_{X}\right) / 2=0.3$.

4. This procedure was repeated 500 times.

5. As the output we get a Monte Carlo approximation to the MISE and MISE*.

Table 1 shows results for both $m(\cdot)$ and $m^{*}(\cdot)$ for the function $m(x)=\sin (2 \pi x)$ and bandwidths $h=0.05, h=1$, respectively. Note that in this case $m=m^{*}$. Even for a relatively small sample size, we may observe that MISE* for the shape function remains constant for either choice of the bandwidth $(h=0.05$ or $h=1$ ). On the other hand, for the small bandwidth $h=0.05$ we observe that MISE for $m(\cdot)$ stays constant up to $d=0.25$, but it grows almost immediately for $h=1$. Furthermore, LRD starts to dominate earlier for the larger bandwidth. This is in line with Propositions 2.5 and 2.7. Next (Table 2), we repeated this experiment with dependent predictors, choosing $d_{X}:=\left(1-\alpha_{X}\right) / 2=0.3$. By comparing both tables, note that there is a little influence of LRD of predictors on MISE for $m(\cdot)$. This is still in line with the Proposition 2.5. On the other hand, for $m^{*}(\cdot)$ estimation, Proposition 2.7 suggests that LRD of predictors should contribute. It does not seem to be the case here, however, or simulation studies suggest that MISE $^{*}$ depends on the constant $\mathrm{E}^{2}\left[m\left(X_{1}\right) X_{1}\right]$, as indicated by our theoretical results.

Finally, based on 500 simulations, we computed averaged values of CV criterion with optimally chosen $h$. Table 3 indicates that LRD influences CV almost immediately, which is once again in line with our theoretical results. 


\begin{tabular}{||c||c|c|c|c||}
\hline \hline \multicolumn{1}{||c||}{$d$} & \multicolumn{2}{c|}{$h=0.05$} & \multicolumn{2}{c||}{$h=1$} \\
\hline & MISE & MISE & MISE & MISE $^{*}$ \\
\hline 0 & 0.3995014 & 0.3983940 & 0.5087492 & 0.5016983 \\
0.05 & 0.3932386 & 0.3870820 & 0.5104933 & 0.5011370 \\
0.10 & 0.3859548 & 0.3791453 & 0.5136161 & 0.5042601 \\
0.15 & 0.3780757 & 0.3724782 & 0.5163214 & 0.5030991 \\
0.20 & 0.4095343 & 0.3910381 & 0.5255956 & 0.5026314 \\
0.25 & 0.4050078 & 0.3850845 & 0.5322871 & 0.5027839 \\
0.30 & 0.4242228 & 0.3794874 & 0.5494079 & 0.5019103 \\
0.35 & 0.4493550 & 0.3940342 & 0.5975138 & 0.5044394 \\
0.40 & 0.5848862 & 0.3805059 & 0.6432639 & 0.5016800 \\
0.45 & 0.8899494 & 0.3822220 & 0.8908165 & 0.5038973 \\
\hline \hline
\end{tabular}

Table 1: MISE for some values of the dependence parameter $d$ and i.i.d. predictors.

\begin{tabular}{||c||cc||}
\hline \hline \multicolumn{1}{c||}{$d$} & \multicolumn{2}{c||}{$h=0.05$} \\
\hline & MISE & MISE* $^{*}$ \\
\hline 0 & 0.4021701 & 0.3963913 \\
0.05 & 0.4454991 & 0.4385924 \\
0.10 & 0.4337356 & 0.4227792 \\
0.15 & 0.4281452 & 0.4225283 \\
0.20 & 0.4262649 & 0.4121518 \\
0.25 & 0.4436659 & 0.4137239 \\
0.30 & 0.4417674 & 0.4049835 \\
0.35 & 0.4948932 & 0.4307672 \\
0.40 & 0.5673097 & 0.4146042 \\
0.45 & 0.8217908 & 0.4258788 \\
\hline \hline
\end{tabular}

Table 2: MISE for some values of the dependence parameter $d$ and LRD predictors.

\section{Examples}

In this section we present some examples, which show that our conditions are easy to verify for very different long memory processes.

Unless specified otherwise, $\left\{Z, Z_{i}, i \geq 1\right\}$ and $\left\{\eta, \eta_{i},-\infty<i<\infty\right\}$ will be sequences of centered i.i.d. random variables with $\mathrm{E}\left[Z_{1}^{1}\right]=\mathrm{E}\left[\eta_{1}^{2}\right]=1$. More detailed description of most of the models below, together with stationarity and moment conditions, can be found in [9].

Example 4.1 (Linear processes). Assume (E2). Then we have

$$
\mathrm{E}\left[\varepsilon_{i} \mid \mathcal{H}_{i-1}\right]=\sum_{k=1}^{\infty} c_{k} \eta_{i-k}+\mathrm{E}\left[\eta_{i} \mid \mathcal{H}_{i-1}\right]=: \varepsilon_{i, i-1}+0
$$




\begin{tabular}{||c||c||}
\hline \hline $\mathrm{d}$ & $\mathrm{CV}$ \\
\hline 0 & 0.2912500 \\
0.05 & 0.2921772 \\
0.10 & 0.2960790 \\
0.15 & 0.2972158 \\
0.20 & 0.310086 \\
0.25 & 0.3206689 \\
0.30 & 0.3415160 \\
0.35 & 0.3687273 \\
0.40 & 0.4133771 \\
0.45 & 0.4663486 \\
\hline \hline
\end{tabular}

Table 3: CV for some values of the dependence parameter $d$ and i.i.d. predictors.

and $\left\{\varepsilon_{i, i-1}, i \geq 1\right\}$ is LRD linear process with (up to a constant) the same limiting behavior as $\left\{\varepsilon_{i}, i \geq 1\right\}$. Consequently,

$$
\sum_{i=1}^{n}\left\{\mathrm{E}\left[\varepsilon_{i} \mid \mathcal{H}_{i-1}\right]-\varepsilon_{i}\right\}=-\sum_{i=1}^{n} \eta_{i}
$$

and

$$
\sum_{i=1}^{n} \mathrm{E}\left[\varepsilon_{i} \mid \mathcal{H}_{i-1}\right]=\sum_{i=1}^{n} \varepsilon_{i, i-1}
$$

By (9) and since $\eta_{i}$ are i.i.d., (A) is automatically fulfilled. Finally, by (10), we conclude that (B2) holds if

$$
h^{5} n^{1-\alpha} \rightarrow 0 .
$$

Note that this condition is much less restrictive than

$$
h n^{1-\alpha} \rightarrow 0,
$$

which is required for (B1) to hold. The latter condition is the same as in [19].

Example 4.2 (Functionals of Linear Processes). Consider the linear process from Example 4.1. Let $T$ be a twice differentiable functional and let

$$
\varepsilon_{i}=T\left(\sum_{k=0}^{\infty} c_{k} \eta_{i-k}\right)=T\left(c_{0} \eta_{i}+\varepsilon_{i, i-1}\right) .
$$

Consider the same assumptions as in Example 4.1. Additionally, we assume that $\mathrm{E}\left[T\left(\varepsilon_{1}\right)\right]=0$.

Let $f_{\eta}$ be the density of $\eta_{1}$. Then, by considering two terms of Taylor 
expansion (which is enough for a quadratic functional),

$$
\begin{aligned}
\mathrm{E} & {\left[\varepsilon_{i} \mid \mathcal{H}_{i-1}\right]=\int T\left(u+\varepsilon_{i, i-1}\right) f_{\eta}(u) d u } \\
& =\int T(u) f_{\eta}(u) d u+\varepsilon_{i, i-1} \int T^{\prime}\left(c_{0} u\right) f_{\eta}(u) d u+\frac{1}{2} \varepsilon_{i, i-1}^{2} \int T^{\prime \prime}(u) f_{\eta}(u) d u \\
& =\mathrm{E}\left[T\left(\eta_{1}\right)\right]+\mathrm{E}\left[T^{\prime}\left(\eta_{1}\right)\right] \varepsilon_{i, i-1}+\frac{1}{2} \mathrm{E}\left[T^{\prime \prime}\left(\eta_{1}\right)\right] \varepsilon_{i, i-1}^{2}
\end{aligned}
$$

and

$$
\varepsilon_{i}=T\left(\eta_{i}\right)+T^{\prime}\left(\eta_{i}\right) \varepsilon_{i, i-1}+\frac{1}{2} T^{\prime \prime}\left(\eta_{i}\right) \varepsilon_{i, i-1}^{2} .
$$

For simplicity take $T(u)=u^{2}-\mathrm{E}\left[\eta_{1}^{2}+\varepsilon_{1,0}^{2}\right]$ and assume that the density $f_{\eta}$ is symmetric. Then $\mathrm{E}\left[T\left(\eta_{1}\right)\right]=\mathrm{E}\left[\varepsilon_{i, i-1}^{2}\right]=-\sum_{k=1}^{\infty} c_{k}^{2}, \mathrm{E}\left[T^{\prime}\left(c \eta_{1}\right)\right]=2 \mathrm{E}\left[\eta_{1}\right]=0$. Consequently,

$$
\operatorname{Var}\left(\sum_{i=1}^{n} \mathrm{E}\left[\varepsilon_{i} \mid \mathcal{H}_{i-1}\right]\right)=\operatorname{Var}\left(\sum_{i=1}^{n}\left(\varepsilon_{i, i-1}^{2}-\mathrm{E}\left[\varepsilon_{i, i-1}^{2}\right]\right)\right) \sim O\left(n^{2-2 \alpha} \vee n\right) .
$$

We conclude that (B2) holds for all $\alpha>1 / 2$ or if $n^{1-2 \alpha} h^{5} \rightarrow 0$, whereas (B1) holds when $\alpha>1 / 2$ or if $n^{1-2 \alpha} h \rightarrow 0$.

Moreover,

$$
\mathrm{E}\left[\varepsilon_{i} \mid \mathcal{H}_{i-1}\right]-\varepsilon_{i}=-\eta_{i}-2 \eta_{i} \varepsilon_{i, i-1}
$$

and thus (A) holds since $\eta_{i}, i \geq 1$ are i.i.d. and $\eta_{i} \varepsilon_{i, i-1}, i \geq 1$ are uncorrelated.

Similar consideration can be carried out for any functional $T$, in particular, for $T(u)=|u|^{\delta}-\mathrm{E}\left[\left|\varepsilon_{1}\right|^{\delta}\right]$. The set of parameters for which (B2) and (B1) hold depends on the so-called power rank of $T$ (see [14] for more details). If the power rank is 1, the (B2) and (B1) hold for $\alpha, h$ as in Example 4.1, if the power rank is 2 , then the conditions are fulfilled for $\alpha, h$ as in case of quadratic functional discussed above.

Example 4.3 (FARIMA-GARCH processes). Assume that

$$
\varepsilon_{i}=(1-B)^{-d} \phi^{-1}(B) \psi(B) \eta_{i}
$$

where $\eta_{i}=Z_{i} h_{i}^{1 / 2}$ and $h_{i}$ is $\operatorname{GARCH}(r, s)$,

$$
h_{i}=a_{0}+\sum_{j=1}^{r} a_{j} \eta_{i-j}^{2}+\sum_{k=1}^{s} \beta_{k} h_{i-k} .
$$

Here, $B$ is the backshift operator, $\psi$ and $\phi$ are polynomials in $B$ and $d \in$ $(-1 / 2,1 / 2)$. Note that under appropriate stationarity conditions the FARIMAGARCH process can be written as the linear process in (E2), where $c_{k} \sim C k^{-\beta}$, $\beta=1-d$ (we refer to [1] for more details).

Let $\mathcal{H}_{i-1}=\sigma\left(\eta_{i}, Z_{i}, \eta_{i-1}, Z_{i-1} \ldots\right)$. Then $\mathrm{E}\left[\eta_{i} \mid \mathcal{H}_{i-1}\right]=\mathrm{E}\left[Z_{i}\right] \mathrm{E}\left[h_{i}^{1 / 2} \mid \mathcal{H}_{i-1}\right]=$ 0. Consequently, as in Example 4.1 (see (8)), (A) holds for all $d \in(-1 / 2,1 / 2)$, since $\eta_{i}$ are uncorrelated. Furthermore, (B2) and (B1) hold if $n^{2 d} h^{5} \rightarrow 0$ and $n^{2 d} h \rightarrow 0$, respectively, on account of [1, Theorem 3]. 
Example 4.4 (Antipersistent errors). If in Example $4.3 d \in(-1 / 2,0)$, then we have antipersistence and (B2) is always fulfilled. Consequently, in case of antipersistent errors the correct scaling for the estimator of both $m$ and $m^{*}$ is always $\sqrt{n h}$. Note that in case of fixed-design regression antipersistency may improve convergence beyond i.i.d. rates, see e.g. [1], [2].

Example 4.5 (Stochastic volatility). Let $T$ and $W$ be real-valued functionals. Define

$$
\varepsilon_{i}^{*}=Z_{i} R_{i}, \quad R_{i}=W\left(a+\sum_{k=1}^{\infty} c_{k} \eta_{i-k}\right), \quad a>0 .
$$

Let

$$
\varepsilon_{i}=T\left(\varepsilon_{i}^{*}\right)-\mathrm{E}\left[T\left(\varepsilon_{i}^{*}\right)\right]
$$

and $\mathcal{H}_{i}=\sigma\left(\eta_{i}, Z_{i}, \eta_{i-1}, Z_{i-1}, \ldots\right)$. Note that $R_{i}$ is $\mathcal{H}_{i-1}$-measurable. For simplicity, assume that $T(u v)=T(u) T(v)$, which applies e.g. to polynomials $T(u)=|u|^{\delta}$. Then

$$
\begin{aligned}
\sum_{i=1}^{n} \mathrm{E}\left[\varepsilon_{i} \mid \mathcal{H}_{i-1}\right] & =\mathrm{E}[T(Z)] \sum_{i=1}^{n}\left\{\mathrm{E}\left[T\left(R_{i}\right) \mid \mathcal{H}_{i-1}\right]-\mathrm{E}\left[T\left(R_{i}\right)\right]\right\} \\
& =\mathrm{E}[T(Z)] \sum_{i=1}^{n}\left\{T\left(R_{i}\right)-\mathrm{E}\left[T\left(R_{i}\right)\right]\right\}
\end{aligned}
$$

Thus, if $c_{k} \sim k^{-(\alpha+1) / 2}, \alpha \in(0,1)$, the conditions for (B2) and (B1) are the same as for nonlinear transformations of linear processes in Example 4.2 by substituting $T \rightarrow T \circ W$.

Furthermore

$$
\sum_{i=1}^{n}\left\{\mathrm{E}\left[\varepsilon_{i} \mid \mathcal{H}_{i-1}\right]-\varepsilon_{i}\right\}=\sum_{i=1}^{n} T\left(R_{i}\right)\left\{\mathrm{E}[T(Z)]-T\left(Z_{i}\right)\right\}=: \sum_{i=1}^{n} T\left(R_{i}\right) U_{i} .
$$

Note that $T\left(R_{i}\right) U_{i}, i \geq 1$, are uncorrelated, thus (A) is always fulfilled.

If $\varepsilon_{i}=\varepsilon_{i}^{*}=Z_{i} R_{i}$, then $\mathrm{E}\left[\varepsilon_{i} \mid \mathcal{H}_{i-1}\right]=0$ and since the random variables $\varepsilon_{i}, i \geq 1$ are uncorrelated, $\sum_{i=1}^{n} \varepsilon_{i}=O_{P}(\sqrt{n})$. Thus, the memory parameter $\alpha$ has no influence on the asymptotic behavior of neither $\hat{m}_{h}$ nor $\hat{m}_{h}^{*}$.

Example 4.6 (LARCH processes). Define

$$
\varepsilon_{i}^{*}=Z_{i} R_{i}, \quad R_{i}=a+\sum_{k=1}^{\infty} c_{k} \varepsilon_{i-k}^{*}, \quad a>0
$$

and assume that $\sum_{k=1}^{\infty} c_{k}^{2}<1, c_{k} \sim k^{-(\alpha+1) / 2}, \alpha \in(0,1)$. Let

$$
\varepsilon_{i}=T\left(\varepsilon_{i}^{*}\right)-\mathrm{E}\left[T\left(\varepsilon_{i}^{*}\right)\right]
$$


and $\mathcal{H}_{i}=\sigma\left(\varepsilon_{i}^{*}, Z_{i}, \epsilon_{i-1}^{*}, Z_{i-1}, \ldots\right)$. The random variable $R_{i}$ is $\mathcal{H}_{i-1}$-measurable. As in Example 4.5, assume that $T(u v)=T(u) T(v)$ so that

$$
\sum_{i=1}^{n}\left\{\mathrm{E}\left[\varepsilon_{i} \mid \mathcal{H}_{i-1}\right]-\varepsilon_{i}\right\}=\sum_{i=1}^{n} T\left(R_{i}\right)\left\{\mathrm{E}[T(Z)]-T\left(Z_{i}\right)\right\}=: \sum_{i=1}^{n} T\left(R_{i}\right) U_{i} .
$$

and $T\left(R_{i}\right) U_{i}, i \geq 1$ are uncorrelated, thus (A) is always fulfilled. Furthermore,

$$
\sum_{i=1}^{n} \mathrm{E}\left[\varepsilon_{i} \mid \mathcal{H}_{i-1}\right]=\mathrm{E}[T(Z)] \sum_{i=1}^{n}\left\{T\left(R_{i}\right)-\mathrm{E}\left[T\left(R_{i}\right)\right]\right\} .
$$

Although this expression has the same form as in Example 4.5, $R_{i}$ is not a linear process based on i.i.d. random variables. Nevertheless, from [3, Theorem 1.1] we conclude that if $T$ is twice differentiable, and $\mathrm{E}\left[R_{1} T\left(R_{1}\right)\right] \neq 0$, then the scaling factor for the latter sum is the same as for linear processes in Example 4.1. Thus, for the conditions (B2) and (B1) to be fulfilled, we need (11) and (12), respectively.

\section{Proofs}

In the proofs we apply a concept of martingale approximation and Hermite expansion. In the context of nonparametric estimation the first method was introduced in [19] and [17], the latter one is a standard tool in LRD setting, see e.g. [18].

Let us note that under the regularity assumptions we have:

$$
\mathrm{E}\left[K_{h}\left(x-X_{1}\right)\right]=h f(x)+h^{3} \cdot \frac{f^{\prime \prime}(x)}{2} \kappa_{2}+o\left(h^{3}\right),
$$

We may write

$$
\hat{m}_{h}(x)-m_{n}(x)=\frac{\hat{f}_{h}(x)}{f(x)}\left(\hat{m}_{h}(x)-m_{n}(x)\right)+\left(\hat{m}_{h}(x)-m_{n}(x)\right) \frac{\left(f(x)-\hat{f}_{h}(x)\right)}{f(x)} .
$$

Since $\hat{f}_{h}$ is the consistent estimator of $f$, it suffices to study the first part only. Decompose

$$
\begin{gathered}
\frac{\hat{f}_{h}(x)}{f(x)}\left(\hat{m}_{h}(x)-m_{n}(x)\right)=\frac{1}{n h f(x)} \sum_{i=1}^{n} K_{h}\left(x-X_{i}\right)\left(m\left(X_{i}\right)-m_{n}(x)\right) \\
+\frac{1}{n h f(x)} \sum_{i=1}^{n} K_{h}\left(x-X_{i}\right) \varepsilon_{i}=: N_{n}(x)+N_{n}^{\prime}(x) .
\end{gathered}
$$


The parts $N_{n}(\cdot)$ and $N_{n}^{\prime}(\cdot)$ are decomposed further as follows:

$$
\begin{aligned}
N_{n}(x) & =\frac{1}{f(x)}\left(m(x)-m_{n}(x)\right)\left(\hat{f}_{h}(x)-\mathrm{E}\left[\hat{f}_{h}(x)\right]\right) \\
& +\frac{1}{n h f(x)} \sum_{i=1}^{n}\left(K_{h}\left(x-X_{i}\right)\left(m\left(X_{i}\right)-m(x)\right)-\mathrm{E}\left[K_{h}\left(x-X_{i}\right)\left(m\left(X_{i}\right)-m(x)\right) \mid \mathcal{X}_{i-1}\right]\right) \\
& +\frac{1}{n h f(x)} \sum_{i=1}^{n}\left(\mathrm{E}\left[K_{h}\left(x-X_{i}\right)\left(m\left(X_{i}\right)-m(x)\right) \mid \mathcal{X}_{i-1}\right]-\mathrm{E}\left[K_{h}\left(x-X_{i}\right)\left(m\left(X_{i}\right)-m(x)\right)\right]\right) \\
=: & N_{n, 1}(x)+N_{n, 0}(x)+N_{n, 2}(x) .
\end{aligned}
$$

Likewise,

$$
\begin{aligned}
N_{n}^{\prime}(x) & =\frac{1}{n h f(x)} \sum_{i=1}^{n} K_{h}\left(x-X_{i}\right) \varepsilon_{i}= \\
= & \frac{1}{n h f(x)} \sum_{i=1}^{n}\left(K_{h}\left(x-X_{i}\right) \varepsilon_{i}-\mathrm{E}\left[K_{h}\left(x-X_{i}\right) \varepsilon_{i} \mid \mathcal{X}_{i-1} \vee \mathcal{H}_{i-1}\right]\right) \\
& +\frac{1}{n h f(x)} \sum_{i=1}^{n} \mathrm{E}\left[K_{h}\left(x-X_{i}\right) \varepsilon_{i} \mid \mathcal{X}_{i-1} \vee \mathcal{H}_{i-1}\right]=: M_{n}(x)+D_{n}(x)
\end{aligned}
$$

Consequently, for $\hat{m}_{h}(x)-m_{n}(x)$ we have the following decomposition:

$$
\frac{\hat{f}_{h}(x)}{f(x)}\left(\hat{m}_{h}(x)-m_{n}(x)\right)=N_{n, 0}(x)+N_{n, 1}(x)+N_{n, 2}(x)+M_{n}(x)+D_{n}(x) .
$$

Note that $M_{n}(\cdot)$ and $N_{n, 0}(\cdot)$ are always martingales.

Assume first $(\mathrm{P} 1)$. Then $N_{n, 2}(\cdot) \equiv 0$. Furthermore,

$$
D_{n}(x)=\frac{1}{n h f(x)} \mathrm{E}\left[K_{h}\left(x-X_{1}\right)\right] \sum_{i=1}^{n} \mathrm{E}\left[\varepsilon_{i} \mid \mathcal{H}_{i-1}\right] .
$$

Assume now (P2). Let $X_{i, i-1}=\mathrm{E}\left[X_{i} \mid \mathcal{X}_{i-1}\right]=\sum_{k=1}^{\infty} a_{k} \zeta_{i-k}$. Let $f_{\zeta_{i}}(\cdot)$ be the density of $\zeta_{i}$. Let $\gamma^{2}=\operatorname{Var}\left(X_{1,0}\right)$, write $Z_{i}=X_{i, i-1} / \gamma$ and note that $Z_{i}$ is independent of $\zeta_{i}$. Let $H_{q}(\cdot)$ be the $q$ th Hermite polynomial. Applying the Hermite expansion we represent $N_{n, 2}(x)$ as

$$
N_{n, 2}(x)=\frac{1}{n h f(x)} \sum_{q=1}^{\infty} \frac{1}{q !} \sum_{i=1}^{n} H_{q}\left(Z_{i}\right) \int L(q ; u) f_{\zeta_{1}}(u) d u,
$$

where

$$
L(q ; u)=L(q ; u, h, \gamma)=\mathrm{E}\left[K_{h}\left(x-\left(u+\gamma Z_{1}\right)\right)\left(m\left(u+\gamma Z_{1}\right)-m(x)\right) H_{q}\left(Z_{1}\right)\right] .
$$


Also,

$$
D_{n}(x)=\frac{1}{n h f(x)} \sum_{q=0}^{\infty} \frac{1}{q !} \sum_{i=1}^{n} H_{q}\left(Z_{i}\right) \mathrm{E}\left[\varepsilon_{i} \mid \mathcal{H}_{i-1}\right] \int J(q ; u) f_{\zeta_{1}}(u) d u,
$$

where

$$
J(q ; u)=J(q ; u, h, \gamma)=\mathrm{E}\left[K_{h}\left(x-\left(u+\gamma Z_{1}\right)\right) H_{q}\left(Z_{1}\right)\right] .
$$

Note that summations in Hermite expansions is from $q=0$, since the expanded functions does not have mean 0 w.r.t. Gaussian density.

Furthermore,

$$
N_{n, 1}(x)=\frac{1}{f(x)}\left(m(x)-m_{n}(x)\right) \frac{1}{n h} \sum_{q=1}^{\infty} \sum_{i=1}^{n} \frac{C(q)}{q !} H_{q}\left(X_{i}\right),
$$

where

$$
C(q)=C(q ; h)=\mathrm{E}\left[K_{h}\left(x-X_{1}\right) H_{q}\left(X_{1}\right)\right] .
$$

As for the shape function $m^{*}$, we write

$$
\hat{m}_{h}^{*}(x)-m^{*}(x)=\left(\hat{m}_{h}^{*}(x)-m_{n}^{*}(x)\right)+\left(m_{n}^{*}(x)-m^{*}(x)\right),
$$

where $m_{n}^{*}(x)=m_{n}(x)-\int m f$. Clearly, $m_{n}^{*}(x)-m^{*}(x)=m_{n}(x)-m(x)$. Let $\hat{\Theta}_{1}=\frac{1}{n} \sum_{i=1}^{n} m\left(X_{i}\right), \hat{\Theta}_{2}=\frac{1}{n} \sum_{i=1}^{n} \varepsilon_{i}$. With this notation we write

$$
\begin{aligned}
\frac{\hat{f}_{h}(x)}{f(x)}\left(\hat{m}_{h}^{*}(x)-m_{n}^{*}(x)\right)=N_{n}(x)+\frac{1}{n h f(x)} \sum_{i=1}^{n} K_{h}\left(x-X_{i}\right)\left(\int m f-\hat{\Theta}_{1}\right) \\
\quad+\frac{1}{n h f(x)} \sum_{i=1}^{n} K_{h}\left(x-X_{i}\right)\left(\varepsilon_{i}-\hat{\Theta}_{2}\right) \\
=\quad N_{n, 0}(x)+M_{n}(x)+\frac{1}{n h f(x)} \sum_{i=1}^{n} K_{h}\left(x-X_{i}\right)\left(\int m f-\hat{\Theta}_{1}\right) \\
\quad+D_{n}(x)-\frac{1}{n h f(x)} \sum_{i=1}^{n} K_{h}\left(x-X_{i}\right) \hat{\Theta}_{2}+N_{n, 1}(x)+N_{n, 2}(x) .
\end{aligned}
$$

The crucial difference between $\hat{m}_{h}(\cdot)$ and $\hat{m}_{h}^{*}(\cdot)$ is that possibly LRD part $D_{n}(x)$ is replaced with

$$
D_{n}(x)-\frac{1}{n h f(x)} \sum_{i=1}^{n} K_{h}\left(x-X_{i}\right) \hat{\Theta}_{2}=: D_{n}(x)-E_{n}(x) .
$$

\subsection{Proof of Propositions 2.1, 2.3}

Recall (15). Since $N_{n, 0}(x)$ and $M_{n}(x)$ are martingales we may easily conclude that

$$
\operatorname{Var}\left(N_{n, 0}(x)\right)=O(h / n), \quad \operatorname{Var}\left(M_{n}(x)\right) \sim \frac{\kappa_{1}}{n h f(x)},
$$


which means that $N_{n, 0}(x)$ is negligible. Furthermore, the martingale part $M_{n}(x)$ may be studied using standard tools (see the proof below in Section 5.1.1).

Lemma 5.1. Under the conditions of Proposition 2.1,

$$
\sqrt{\frac{n h}{\kappa_{1}}} \sqrt{f(x)} M_{n}(x) \stackrel{\mathrm{d}}{\rightarrow} N(0,1) .
$$

Under (P1), using (2),

$$
\operatorname{Var}\left[N_{n, 1}(x)\right]=O\left(h^{4}(n h)^{-1}\right)=O\left(h^{3} / n\right),
$$

so that this term is negligible w.r.t. $M_{n}(\cdot)$ as well. Furthermore, using (16) and (13) we have

$$
D_{n}(x)=\left(1+h^{2} \frac{\kappa_{2}}{2} \frac{f^{\prime \prime}(x)}{f(x)}+o\left(h^{2}\right)\right) \frac{1}{n} \sum_{i=1}^{n} \mathrm{E}\left[\varepsilon_{i} \mid \mathcal{H}_{i-1}\right] .
$$

Consequently, under (P1) the result follows form Lemma 5.1 and assumption (B1), which makes $D_{n}(\cdot)$ negligible.

Now, we work under the assumption (P2). Recall (17). We split the stochastic term there as

$$
C(1) \sum_{i=1}^{n} X_{i}+\sum_{q=2}^{\infty} \sum_{i=1}^{n} \frac{C(q)}{q !} H_{q}\left(X_{i}\right) .
$$

Using orthonormality of the Hermite polynomials, the variance of the latter term is

$$
\sum_{i=1}^{n} \sum_{q=2}^{\infty} \frac{C^{2}(q)}{q !} \operatorname{Cov}^{q}\left(X_{1}, X_{i}\right) \leq \sum_{i=1}^{n} \operatorname{Cov}^{2}\left(X_{1}, X_{i}\right)\left\|K_{h}(x-\cdot)\right\|,
$$

where $\|\cdot\|$ stands for $L^{2}$ norm with respect to the Gaussian measure. Since $\left\|K_{h}(x-\cdot)\right\|$ and $C(1) \sim h x f(x)$, we conclude that the leading term in $N_{n, 1}(x)$ is

$$
\frac{1}{f(x)}\left(m(x)-m_{n}(x)\right) \frac{C(1)}{h f(x)} \frac{1}{n} \sum_{i=1}^{n} X_{i} .
$$

This implies

$$
\operatorname{Var}\left(N_{n, 1}(x)\right)=O\left(h^{4} n^{-\alpha_{X}}\right) .
$$

The similar consideration is applied to $D_{n}$ and $N_{n, 2}$. Using the independence of $Z_{1}$ and $\zeta_{1}$, we have

$$
\int J(0 ; u) f_{\zeta_{1}}(u) d u=\mathrm{E}\left[K_{h}\left(x-X_{1}\right)\right]=h f(x)+\frac{1}{2} h^{3} f^{\prime \prime}(x) \int u^{2} K(u) d u+o\left(h^{3}\right) .
$$


This yields

$$
D_{n}(x)=\frac{1}{n} \sum_{i=1}^{n} \mathrm{E}\left[\varepsilon_{i} \mid \mathcal{H}_{i-1}\right]+o_{P}(1) .
$$

The leading terms in $N_{n, 2}(x)$ is

$$
\frac{\mathrm{E}\left[L\left(1 ; \zeta_{1}\right)\right]}{h f(x)} \frac{1}{n} \sum_{i=1}^{n} Z_{i}
$$

which implies

$$
\operatorname{Var}\left(N_{n, 2}(x)\right)=O\left(h^{4} n^{-\alpha_{X}}\right) .
$$

Consequently, under (P2), the result follows from Lemma 5.1 and assumption (B1), which makes $D_{n}(\cdot)$ negligible, together with $(\mathrm{C} 1)$, which makes $N_{n, 1}(x)+N_{n, 2}(\cdot)$ negligible.

We prove now Proposition 2.3 assuming (P2). Recall (18). It was proven before that $N_{n, 0}(x), N_{n, 1}(x)$ and $N_{n, 2}(x)$ are negligible. The first term in the Hermite expansion for $N_{n, 2}^{\prime}(x)$ is

$$
\frac{1}{n h f(x)} \mathrm{E}\left[J\left(0 ; \zeta_{1}\right)\right] \sum_{i=1}^{n} \mathrm{E}\left[\varepsilon_{i} \mid \mathcal{H}_{i-1}\right] .
$$

If $\alpha+\alpha_{X}>1$, then $\sum_{i=1}^{n} \mathrm{E}\left[\varepsilon_{i} \mid \mathcal{H}_{i-1}\right] Z_{i}=O_{P}(\sqrt{n})$, otherwise $\sum_{i=1}^{n} \mathrm{E}\left[\varepsilon_{i} \mid \mathcal{H}_{i-1}\right] Z_{i}=$ $O_{P}\left(n^{1-\left(\alpha+\alpha_{X}\right) / 2}\right)$. Since for $q \geq 0, \mathrm{E}\left[J\left(q ; \zeta_{1}\right)\right]=O(h)$, we conclude that $D_{n}(x)$ can be written as

$$
\frac{1}{n h f(x)} \mathrm{E}\left[J\left(0 ; \zeta_{1}\right)\right] \sum_{i=1}^{n} \mathrm{E}\left[\varepsilon_{i} \mid \mathcal{H}_{i-1}\right]+O_{P}\left(n^{-\left(\alpha+\alpha_{X}\right) / 2}\right)+O_{P}\left(n^{-1 / 2}\right) .
$$

The first two terms in the Hermite expansion for $E_{n}(x)$ are

$$
\Theta_{2} \frac{1}{h f(x)} \mathrm{E}\left[K_{h}\left(x-X_{1}\right)\right]+\Theta_{2} \frac{1}{h f(x)} \mathrm{E}\left[K_{h}\left(x-X_{1}\right) X_{1}\right] \frac{1}{n} \sum_{i=1}^{n} X_{i} .
$$

Using (22), and $\frac{1}{n} \sum_{i=1}^{n} X_{i}=O_{P}\left(n^{-\alpha_{X} / 2}\right)$, we conclude that the leading terms in the difference $N_{n, 2}^{\prime}(x)-E_{n}(x)$, are

$$
\begin{aligned}
& \frac{1}{n} \sum_{i=1}^{n}\left(\mathrm{E}\left[\varepsilon_{i} \mid \mathcal{H}_{i-1}\right]-\varepsilon_{i}\right) \\
& \quad+O_{P}\left(\frac{h^{2}}{n} \sum_{i=1}^{n} \mathrm{E}\left[\varepsilon_{i} \mid \mathcal{H}_{i-1}\right]\right)+O_{P}\left(n^{-\alpha_{X} / 2} \frac{1}{n} \sum_{i=1}^{n} \mathrm{E}\left[\varepsilon_{i} \mid \mathcal{H}_{i-1}\right]\right) .
\end{aligned}
$$

The first two terms are negligible under the conditions (A) and (B2), respectively. The last term is negligible on account of (4), which is weaker than (C2). Finally,

$$
n^{\alpha_{X} / 2}\left(\int m f-\hat{\Theta}_{1}\right) \stackrel{\mathrm{d}}{\rightarrow} \mathcal{N}\left(0, A_{1}^{2} \mathrm{E}^{2}\left[m\left(X_{1}\right) X_{1}\right]\right),
$$


which makes the term

$$
\frac{1}{n h f(x)} \sum_{i=1}^{n} K_{h}\left(x-X_{i}\right)\left(\int m f-\hat{\Theta}_{1}\right)
$$

negligible on account of condition (4).

\subsubsection{Proof of Lemma 5.1}

Proof of Lemma 5.1. The proof is similar to [19, Lemma 2] and [15, Lemma 3.1]. Let $R_{i}=\left(n h \kappa_{1}\right)^{-1 / 2} K_{h}\left(x-X_{i}\right) \varepsilon_{i} / \sqrt{f(x)}$ and $\bar{R}_{i}=R_{i}-\mathrm{E}\left[R_{i} \mid \mathcal{F}_{i-1}\right]$. From the martingale central limit theorem it suffices to show the Lindeberg condition

$$
\sum_{i=1}^{n} \mathrm{E}\left[\bar{R}_{i}^{2} 1_{\left\{\left|\bar{R}_{i}\right|>\delta\right\}}\right] \rightarrow 0 \text { for each } \delta>0
$$

and convergence of conditional variances

$$
\sum_{i=1}^{n} \mathrm{E}\left[\bar{R}_{i}^{2} \mid \mathcal{F}_{i-1}\right] \stackrel{\mathrm{P}}{\rightarrow} 1
$$

Let $f_{\varepsilon}$ be the density of $\varepsilon_{1}$. As for the Lindeberg condition we have

$$
\begin{aligned}
& \sum_{i=1}^{n} \mathrm{E}\left[\bar{R}_{i}^{2} 1_{\left\{\left|\bar{R}_{i}\right|>\delta\right\}}\right] \leq 4 \sum_{i=1}^{n} \mathrm{E}\left[R_{i}^{2} 1_{\left\{\left|R_{i}\right|>\delta\right\}}\right] \\
& =C_{0} \frac{1}{n h} \sum_{i=1}^{n} \iint K_{h}^{2}(x-u) f(u) v^{2} g_{\varepsilon_{1}}(v) 1_{\left\{|v|>C_{1} \delta \sqrt{n h}\right\}} \\
& \quad \leq C_{2} \frac{1}{n} \sum_{i=1}^{n} \mathrm{E}\left[\varepsilon_{i}^{2} 1_{\left\{\left|\varepsilon_{i}\right|>C_{1} \delta \sqrt{n h}\right\}}\right] \rightarrow 0,
\end{aligned}
$$

where $C_{0}=1 /\left(\kappa_{1} f(x)\right), C_{1}=\left(\sqrt{C_{0}} \sup K(x)\right)^{-1}$ and $C_{2}=C_{0} \int K^{2}$.

As for the conditional variances note first that

$$
\mathrm{E}\left[\bar{R}_{i}^{2} \mid \mathcal{F}_{i-1}\right]=\mathrm{E}\left[R_{i}^{2} \mid \mathcal{F}_{i-1}\right]-\mathrm{E}\left[\left(\mathrm{E}\left[R_{i} \mid \mathcal{F}_{i-1}\right]\right)^{2}\right]
$$

and note that the second term is of smaller order than the first one. Now,

$$
\begin{aligned}
& \sum_{i=1}^{n}\left\{\mathrm{E}\left[R_{i}^{2} \mid \mathcal{F}_{i-1}\right]-\mathrm{E}\left[R_{i}^{2}\right]\right\}=\frac{1}{n h f(x) \kappa_{1}} \mathrm{E}\left[K_{h}^{2}\left(x-X_{1}\right)\right] \sum_{i=1}^{n}\left\{\mathrm{E}\left[\varepsilon_{i}^{2} \mid \mathcal{F}_{i-1}\right]-\mathrm{E}\left[\varepsilon_{i}^{2}\right]\right\} \\
& =\left(\frac{1}{f(x) \kappa_{1}} \int K^{2}(v) f(x-v h) d v\right) \frac{1}{n} \sum_{i=1}^{n}\left\{\mathrm{E}\left[\varepsilon_{i}^{2} \mid \mathcal{F}_{i-1}\right]-\mathrm{E}\left[\varepsilon_{i}^{2}\right]\right\} .
\end{aligned}
$$

Now, the deterministic term in the bracket is asymptotically equal to 1 . The second part converges to 0 in probability from ergodicity. Consequently, the expression (26) is proven. 


\subsection{Proof of Propositions 2.5 and 2.7}

Proof. Recall (2), (14) and (15). Under (P1), the result of Proposition 2.5 follows from (19), (20). Under (P2), we use the expansion with (19), (23), (21), (24).

As for Proposition 2.7, note that all considerations for $N_{n, 2}^{\prime}(x)-E_{n}(x)$ leading to (25) are in fact in $L^{2}$. Therefore, on account of (5)

$$
\operatorname{Var}\left(N_{n, 2}^{\prime}(x)-E_{n}(x)\right) \sim h^{4} n^{-\alpha}+O\left(n^{-\left(\alpha+\alpha_{X}\right)}\right) .
$$

The first part is of course negligible w.r.t. the bias term.

Moreover, writing

$$
\begin{aligned}
& \frac{1}{n h f(x)} \sum_{i=1}^{n} K_{h}\left(x-X_{i}\right)\left(\int m f-\hat{\Theta}_{1}\right)=\frac{1}{h f(x)} \mathrm{E}\left[K_{h}\left(x-X_{1}\right)\right]\left(\int m f-\hat{\Theta}_{1}\right) \\
& \quad+\frac{1}{n h f(x)} \sum_{i=1}^{n}\left(K_{h}\left(x-X_{i}\right)-\mathrm{E}\left[K_{h}\left(x-X_{1}\right)\right]\right)\left(\int m f-\hat{\Theta}_{1}\right),
\end{aligned}
$$

we obtain that the variance contribution of this term is $A_{1}^{2} \mathrm{E}^{2}\left[m\left(X_{1}\right) X_{1}\right] n^{-\alpha_{X}}$.

\subsection{Cross validation properties}

Under the condition (E2) one can establish the following moment bounds:

$$
\begin{aligned}
\mathrm{E}\left[\varepsilon_{i}^{2} \varepsilon_{j} \varepsilon_{j^{\prime}}\right] & =O\left(\operatorname{Cov}\left(\varepsilon_{j}, \varepsilon_{j^{\prime}}\right)\right), \\
\mathrm{E}\left[\varepsilon_{j} \varepsilon_{j^{\prime}} \varepsilon_{l} \varepsilon_{l^{\prime}}\right] & =O\left(\mathrm{E}\left[\varepsilon_{j} \varepsilon_{j^{\prime}}\right] \mathrm{E}\left[\varepsilon_{l} \varepsilon_{l^{\prime}}\right]\right), \\
\operatorname{Cov}\left(\varepsilon_{j}^{2}, \varepsilon_{j^{\prime}}^{2}\right) & =O\left(\operatorname{Cov}\left(\varepsilon_{j}, \varepsilon_{j^{\prime}}\right)\right) .
\end{aligned}
$$

\subsubsection{Asymptotic expansion for $\operatorname{ASE}(h)$}

Recall that we work under the condition (P1). Define

$$
R(x)=\frac{1}{n h \hat{f}_{h}(x)} \sum_{j=1}^{n} K_{h}\left(x-X_{j}\right) m\left(X_{j}\right)-m(x) .
$$

Write

$$
\begin{aligned}
\operatorname{ASE}(h) & =I_{21}+I_{22}+I_{23}:=\frac{1}{n^{3} h^{2}} \sum_{i=1}^{n} \frac{1}{\hat{f}_{h}^{2}\left(X_{i}\right)}\left(\sum_{j=1}^{n} K_{h}\left(X_{i}-X_{j}\right) \varepsilon_{j}\right)^{2} \\
& +\frac{1}{n} \sum_{i=1}^{n} R^{2}\left(X_{i}\right)+\frac{2}{n} \sum_{i=1}^{n} R\left(X_{i}\right) \frac{1}{n h \hat{f}_{h}\left(X_{i}\right)} \sum_{j=1}^{n} K_{h}\left(X_{i}-X_{j}\right) \varepsilon_{j} .
\end{aligned}
$$


Let $\rho(x)=(m f)^{\prime \prime}(x)-m(x) f^{\prime \prime}(x)$. Uniformly over $\{x: f(x)>0\}$ we have

$$
R(x)-\frac{h^{2} \kappa_{2}}{2} \frac{\rho(x)}{f(x)}=O\left(h^{4}\left(1+o_{P}(1)\right)\right) .
$$

Using (30) we write the second part as

$$
I_{22}=\kappa_{2}^{2} \frac{h^{4}}{4 n} \sum_{i=1}^{n}\left[\frac{\rho\left(X_{i}\right)}{f\left(X_{i}\right)}\right]^{2}\left(1+o_{P}(1)\right),
$$

so that via $\mathrm{E}\left[\left(\frac{\rho\left(X_{i}\right)}{f\left(X_{i}\right)}\right)^{2}\right]=\int(\rho(x))^{2} / f(x) d x$ we conclude

$$
I_{22}-\kappa_{2}^{2} \frac{h^{4}}{4} \int \frac{\rho^{2}(x)}{f(x)} d x=o_{P}\left(h^{4} / \sqrt{n}\right) .
$$

Now, if $A_{i}, i \geq 1$, are random variables with the same mean, and $\bar{A}_{i}=A_{i}-\mathrm{E}\left[A_{i}\right]$, then we have the following decomposition (which will be used many times):

$$
\sum_{i=1}^{n} A_{i} \varepsilon_{i}=\mathrm{E}\left[A_{1}\right] \sum_{i=1}^{n} \varepsilon_{i}+\sum_{i=1}^{n} \bar{A}_{i} \varepsilon_{i}
$$

Typically, in LRD setting, the first part dominates. Bearing in mind the above decomposition and since $\hat{f}_{h}$ is a consistent estimator of $f$,

$$
\begin{aligned}
I_{23}= & \frac{h^{2}}{n} \frac{1}{n h} \sum_{i=1}^{n} \frac{\rho\left(X_{i}\right)}{f^{2}\left(X_{i}\right)} \sum_{j=1}^{n} K_{h}\left(X_{i}-X_{j}\right) \varepsilon_{j}\left(1+o_{P}(1)\right) \\
= & \mathrm{E}\left[\frac{\rho\left(X_{1}\right)}{f^{2}\left(X_{1}\right)} K_{h}\left(X_{1}-X_{2}\right)\right] \frac{h}{n} \sum_{j=1}^{n} \varepsilon_{j}\left(1+o_{P}(1)\right) \\
& +\frac{h^{2}}{n} \frac{1}{n h} \sum_{i=1}^{n} \sum_{j=1}^{n} \frac{\rho\left(X_{i}\right)}{f^{2}\left(X_{i}\right)} K_{h}\left(X_{i}-X_{j}\right) \varepsilon_{j}\left(1+o_{P}(1)\right) .
\end{aligned}
$$

The second term is negligible w.r.t. to the first one. Noting that

$$
\mathrm{E}\left[\frac{\rho\left(X_{1}\right)}{f^{2}\left(X_{1}\right)} K_{h}\left(X_{1}-X_{2}\right)\right]=h \int \rho(x) d x+O\left(h^{3}\right)
$$

we get

$$
I_{23}=O_{P}\left(\frac{h^{2}}{n} \sum_{j=1}^{n} \varepsilon_{j}\right)
$$


It remains to deal with $I_{21}$. Split it as

$$
\begin{aligned}
\frac{1}{n^{3} h^{2}} \sum_{i=1}^{n} & \frac{1}{f^{2}\left(X_{i}\right)} \sum_{\substack{j, j^{\prime}=1 \\
j \neq j^{\prime}}}^{n} K_{h}\left(X_{i}-X_{j}\right) K_{h}\left(X_{i}-X_{j^{\prime}}\right) \varepsilon_{j} \varepsilon_{j^{\prime}} \\
& +\frac{1}{n h^{2}} \mathrm{E}\left[\frac{1}{f^{2}\left(X_{1}\right)} K_{h}^{2}\left(X_{1}-X_{2}\right) \varepsilon_{1}^{2}\right](1+o(1)) \\
& +\frac{1}{n^{3} h^{2}} \sum_{i, j=1}^{n} \frac{1}{f^{2}\left(X_{i}\right)}\left(K_{h}^{2}\left(X_{i}-X_{j}\right) \varepsilon_{j}^{2}-\mathrm{E}\left[K_{h}^{2}\left(X_{i}-X_{j}\right) \varepsilon_{j}^{2}\right]\right) \\
=: & I_{211}+I_{212}+I_{213} .
\end{aligned}
$$

Clearly,

$$
I_{212}=\frac{1}{n h} \int K^{2}(u) d u+O(h / n) .
$$

To deal with $I_{211}$, define

$$
T_{i}\left(h, X_{j}, X_{j^{\prime}}\right)=\frac{1}{h^{2} f^{2}\left(X_{i}\right)} K_{h}\left(X_{i}-X_{j}\right) K_{h}\left(X_{i}-X_{j^{\prime}}\right),
$$

and note that

$$
\mathrm{E}\left[T_{1}\left(h, X_{2}, X_{3}\right)\right]=1+h^{2} \kappa_{2} \int f^{\prime \prime}+o\left(h^{2}\right)
$$

Split $I_{211}$ as

$$
\frac{\mathrm{E}\left[T_{1}\left(h, X_{2}, X_{3}\right)\right]}{n^{3}} \sum_{i=1}^{n} \sum_{\substack{j, j^{\prime}=1 \\ j \neq j^{\prime}}}^{n} \varepsilon_{j} \varepsilon_{j^{\prime}}+\frac{1}{n^{3}} \sum_{i=1}^{n} \sum_{\substack{j, j^{\prime}=1 \\ j \neq j^{\prime}}}^{n} \overline{T_{i}\left(h, X_{j}, X_{j^{\prime}}\right)} \varepsilon_{j} \varepsilon_{j^{\prime}} .
$$

Variance of the last term is proportional to

$$
\frac{1}{n^{6}} \sum_{i, i^{\prime}} \operatorname{Cov}\left(\sum_{\substack{j, j^{\prime}=1 \\ j \neq j^{\prime}}}^{n} T_{i}\left(h, X_{j}, X_{j^{\prime}}\right), \sum_{\substack{j, j^{\prime}=1 \\ l \neq l^{\prime}}}^{n} T_{i^{\prime}}\left(h, X_{l}, X_{l^{\prime}}\right)\right) \mathrm{E}\left[\varepsilon_{j} \varepsilon_{j^{\prime}} \varepsilon_{l} \varepsilon_{l^{\prime}}\right] .
$$

If all six indices are different than the term vanishes. If the indices $j, j^{\prime}, l, l^{\prime}$ are different, but $i=i^{\prime}$, then via (28) we obtain that the variance contribution is

$$
\frac{1}{n^{6} h^{4}} h^{4} n n^{2-\alpha} n^{2-\alpha}=O\left(1 / n^{1+2 \alpha}\right) .
$$

If $j=l$ and $j^{\prime} \neq l^{\prime}$, together with $i=i^{\prime}$ or $i \neq i^{\prime}$, respectively, then via (27) the variance contribution is, respectively,

$$
\frac{1}{n^{6} h^{4}} n h^{3} n n^{2-\alpha}=O\left(\frac{1}{n^{2+\alpha} h}\right), \quad O\left(\frac{1}{n^{1+\alpha}}\right) .
$$


If $j=l$ and $j^{\prime}=l^{\prime}$, the contribution is $O\left(1 /(n h)^{2}\right)$. Consequently, via (34) and (35), for $I_{211}$ we have

$I_{211} \sim \frac{1+h^{2} \kappa_{2} f^{\prime \prime}}{n^{2}} \sum_{\substack{j, j^{\prime}=1 \\ j \neq j^{\prime}}}^{n} \varepsilon_{j} \varepsilon_{j^{\prime}}+o_{P}\left(\frac{h^{2}}{n^{2}} \sum_{\substack{j, j^{\prime}=1 \\ j \neq j^{\prime}}}^{n} \varepsilon_{j} \varepsilon_{j^{\prime}}\right)+O_{P}\left(\frac{1}{n^{(1+\alpha) / 2}}\right)+O_{P}\left(\frac{1}{n^{1+\alpha / 2} h^{1 / 2}}\right)$.

Now, for $I_{213}$, its variance can be written as

$$
\frac{1}{n^{6} h^{4}} \sum_{j, j^{\prime}=1}^{n} \operatorname{Cov}\left(\varepsilon_{j}^{2}, \varepsilon_{j^{\prime}}^{2}\right) \mathrm{E}\left[\sum_{i, i^{\prime}=1}^{n} \frac{1}{f^{2}\left(X_{i}\right)} K_{h}^{2}\left(X_{i}-X_{j}\right) \frac{1}{f^{2}\left(X_{i^{\prime}}\right)} K_{h}^{2}\left(X_{i^{\prime}}-X_{j^{\prime}}\right)\right] .
$$

Using (29), one can verify that the above expression is

$$
\frac{O(1)}{n^{6} h^{4}}\left(n^{2-2 \alpha} \vee n\right) n^{2} h^{2}=O\left(\frac{1}{n^{2+2 \alpha} h^{2}}\right)+O\left(\frac{1}{n^{3} h^{2}}\right) .
$$

Via (33), (36), (37),

$$
\begin{aligned}
I_{21} & -\frac{1}{n h} \int K^{2}(u) d u-\frac{1}{n^{2}} \sum_{\substack{j, j^{\prime}=1 \\
j \neq j^{\prime}}}^{n} \varepsilon_{j} \varepsilon_{j^{\prime}}-\int f^{\prime \prime} \frac{\kappa_{2} h^{2}}{n^{2}} \sum_{\substack{j, j^{\prime}=1 \\
j \neq j^{\prime}}}^{n} \varepsilon_{j} \varepsilon_{j^{\prime}}=O_{P}\left(\frac{h}{n}\right) \\
& +o_{P}\left(\frac{h^{2}}{n^{2}} \sum_{\substack{j, j^{\prime}=1 \\
j \neq j^{\prime}}}^{n} \varepsilon_{j} \varepsilon_{j^{\prime}}\right)+O_{P}\left(\frac{1}{n^{(1+\alpha) / 2}}\right)+O_{P}\left(\frac{1}{n^{1+\alpha / 2} h^{1 / 2}}\right)+O_{P}\left(\frac{1}{n^{1+\alpha} h}\right) .
\end{aligned}
$$

Furthermore,

$$
\mathrm{E}\left[\frac{1}{n^{2}} \sum_{\substack{j, j^{\prime}=1 \\ j \neq j^{\prime}}}^{n} \varepsilon_{j} \varepsilon_{j^{\prime}}\right] \sim C_{1}^{2} n^{-\alpha}
$$

Combining this with (31) and (32), we have

$$
\begin{gathered}
\operatorname{ASE}(h)-\frac{1}{n h} \int K^{2}(u) d u-\kappa_{2}^{2} \frac{h^{4}}{4} \int \frac{\rho^{2}(x)}{f(x)} d x-C_{1}^{2} n^{-\alpha}-C_{1}^{2} \kappa_{2} h^{2} n^{-\alpha} \int f^{\prime \prime}= \\
\quad o_{P}\left(\frac{h^{2}}{n^{2}} \sum_{\substack{j, j^{\prime}=1 \\
j \neq j^{\prime}}}^{n} \varepsilon_{j} \varepsilon_{j^{\prime}}\right)+O_{P}\left(\frac{h^{2}}{n} \sum_{j=1}^{n} \varepsilon_{j}\right)+O_{P}\left(\frac{h}{n}\right)+O_{P}\left(\frac{1}{n^{(1+\alpha) / 2}}\right) \\
+O_{P}\left(\frac{1}{n^{1+\alpha / 2} h^{1 / 2}}\right)+O_{P}\left(\frac{1}{n^{1+\alpha} h}\right) .
\end{gathered}
$$

Consequently, (6) is proven. 


\subsubsection{Asymptotic expansion for $\mathrm{CV}(h)$}

Recall that

$$
\mathrm{CV}(h):=\frac{1}{n} \sum_{i=1}^{n}\left(Y_{i}-\hat{m}_{i, h}\left(X_{i}\right)\right)^{2} .
$$

Note that we may write

$$
\begin{aligned}
\mathrm{CV}(h) & =\frac{1}{n} \sum_{i=1}^{n}\left(Y_{i}-\hat{m}_{h}\left(X_{i}\right)\right)^{2}+O_{P}(1 /(n h)) \\
& =\operatorname{ASE}(h)+\frac{1}{n} \sum_{i=1}^{n} \varepsilon_{i}^{2}+\frac{2}{n} \sum_{i=1}^{n} \varepsilon_{i}\left(m\left(X_{i}\right)-\hat{m}_{h}\left(X_{i}\right)\right)+O_{P}(1 /(n h)) .
\end{aligned}
$$

Here, $O_{P}(1 /(n h))$ comes from replacing $\hat{m}_{i, h}(\cdot)$ by $\hat{m}_{h}(\cdot)$. The second last term is treated in the very same way as we dealt with $I_{23}$ and $I_{211}$, see (32) and (36), respectively. Therefore, it is

$$
O_{P}\left(\frac{h^{2}}{n} \sum_{j=1}^{n} \varepsilon_{j}+\frac{1}{n^{2}} \sum_{j, j^{\prime}=1}^{n} \varepsilon_{j} \varepsilon_{j^{\prime}}\right) .
$$

Furthermore, $\frac{1}{n^{2}} \sum_{j, j^{\prime}=1}^{n} \varepsilon_{j} \varepsilon_{j^{\prime}}=O_{P}\left(n^{-\alpha}\right)$ and it dominates the term $O_{P}\left(\frac{1}{n^{(1+\alpha) / 2}}\right)$. Consequently, via (38),

$$
\frac{\mathrm{CV}(h)-\operatorname{MISE}_{f}(h)-\frac{1}{n} \sum_{i=1}^{n} \varepsilon_{i}^{2}}{\operatorname{MISE}_{f}\left(h_{\mathrm{opt}}\right)}=\frac{1}{\operatorname{MISE}_{f}\left(h_{\mathrm{opt}}\right)} \frac{1}{n^{2}} \sum_{j, j^{\prime}=1}^{n} \varepsilon_{j} \varepsilon_{j^{\prime}}+o_{p}(1),
$$

uniformly over $\left[B_{1} h_{\mathrm{opt}}, B_{2} h_{\mathrm{opt}}\right]$.

\section{Acknowledgement}

The work of the first author was supported by a NSERC (Natural Sciences and Engineering Research Council of Canada) grant. The work of the second author was conducted while being a Postdoctoral Fellow at the University of Ottawa.

\section{References}

[1] Jan Beran and Yuanhua Feng. Local polynomial estimation with a FARIMA-GARCH error process. Bernoulli, 7(5):733-750, 2001.

[2] Jan Beran and Yuanhua Feng. Local polynomial fitting with longmemory, short-memory and antipersistent errors. Ann. Inst. Statist. Math., $54(2): 291-311,2002$.

[3] István Berkes and Lajos Horváth. Asymptotic results for long memory LARCH sequences. Ann. Appl. Probab., 13(2):641-668, 2003. 
[4] Bing Cheng and P. M. Robinson. Semiparametric estimation from time series with long-range dependence. J. Econometrics, 64(1-2):335-353, 1994.

[5] Gerda Claeskens and Peter Hall. Effect of dependence on stochastic measures of accuracy of density estimators. Ann. Statist., 30(2):431-454, 2002.

[6] Sándor Csörgő and Jan Mielniczuk. Random-design regression under longrange dependent errors. Bernoulli, 5(2):209-224, 1999.

[7] Sándor Csörgő and Jan Mielniczuk. The smoothing dichotomy in randomdesign regression with long-memory errors based on moving averages. Statist. Sinica, 10(3):771-787, 2000.

[8] Sam Efromovich. How to overcome the curse of long-memory errors. IEEE Trans. Inform. Theory, 45(5):1735-1741, 1999.

[9] Liudas Giraitis, Remigijus Leipus, and Donatas Surgailis. Recent advances in ARCH modelling. In Long memory in economics, pages 3-38. Springer, Berlin, 2007.

[10] Peter Hall and Jeffrey D. Hart. Convergence rates in density estimation for data from infinite-order moving average processes. Probab. Theory Related Fields, 87(2):253-274, 1990.

[11] Peter Hall and Jeffrey D. Hart. Nonparametric regression with long-range dependence. Stochastic Process. Appl., 36(2):339-351, 1990.

[12] Peter Hall, Soumendra Nath Lahiri, and Jörg Polzehl. On bandwidth choice in nonparametric regression with both short- and long-range dependent errors. Ann. Statist., 23(6):1921-1936, 1995.

[13] Peter Hall, Soumendra Nath Lahiri, and Young K. Truong. On bandwidth choice for density estimation with dependent data. Ann. Statist., 23(6):2241-2263, 1995.

[14] Hwai-Chung Ho and Tailen Hsing. Limit theorems for functionals of moving averages. Ann. Probab., 25(4):1636-1669, 1997.

[15] Rafał Kulik. Nonparametric deconvolution problem for dependent sequences. Electron. J. Statist., 2:722-740, 2008.

[16] Rafał Kulik and Marc Raimondo. Wavelet regression in random design with heteroscedastic dependent errors. Ann. Statist., 37:3396-3430, 2009.

[17] Jan Mielniczuk and Wei Biao Wu. On random-design model with dependent errors. Statist. Sinica, 14(4):1105-1126, 2004.

[18] Murad S. Taqqu. Fractional Brownian motion and long-range dependence. In Theory and applications of long-range dependence, pages 5-38. Birkhäuser Boston, Boston, MA, 2003. 
[19] Wei Biao Wu and Jan Mielniczuk. Kernel density estimation for linear processes. Ann. Statist., 30(5):1441-1459, 2002.

[20] Yuhong Yang. Nonparametric regression with dependent errors. Bernoulli, 7(4):633-655, 2001. 